\title{
An extensive grid of mass fluxes for Galactic 0 stars
}

\author{
L. B. Lucy
}

\author{
Astrophysics Group, Blackett Laboratory, Imperial College London, Prince Consort Road, London SW7 2AZ, UK \\ e-mail: 1.lucy@imperial.ac.uk
}

Received 13 April 2010 / Accepted 8 September 2010

\section{ABSTRACT}

\begin{abstract}
A previously-described code for constructing moving reversing layers (MRL) is improved by replacing a two-parameter model for $g^{\mathrm{l}}(v)$, the radiative acceleration due to lines, with a flexible non-parametric description, thus allowing a greater degree of dynamical consistency in modelling turbulent transonic flow in the outer atmospheric layers of O stars. With this new code, mass fluxes $J$ are computed at fifty-seven points in $\left(T_{\mathrm{eff}}, g\right)$-space. Specifically, $J$ 's are computed for all Lanz-Hubeny (2003) NLTE atmospheres with $T_{\text {eff }}(k K) \in(27.5,55)$ and $\log g\left(\mathrm{~cm} \mathrm{~s}^{-2}\right) \leq 4$.5. Differences with widely-used mass-loss formulae are emphasized, and opportunities for differential spectroscopic tests identified.
\end{abstract}

Key words. stars: early-type - stars: mass-loss - stars: winds, outflows

\section{Introduction}

In an earlier paper (Lucy 2007, L07), the moving reversing layer (MRL) theory of Lucy \& Solomon (1970, LS70) was updated by incorporating an extensive line list and improving the treatment of line formation. In addition, the models were, in effect, grafted onto the TLUSTY static NLTE O-star atmospheres of Lanz \& Hubeny (2003) by imposing the TLUSTY emergent continuum flux distribution as the lower radiative boundary condition and by matching ionization fractions at $T \approx 0.75 T_{\text {eff }}$.

The motivation for reviving MRL theory was the conflict between observed and predicted mass loss rates $(\Phi)$, which had led several spectroscopic groups to question the theory of radiatively-driven winds (e.g., Bouret et al. 2005; Fullerton et al. 2006). But, as emphasized in L07, the $\Phi$ 's being tested were not obtained by solving the equations governing the dynamics of radiatively-driven winds but are the values $\Phi_{V}$ derived by Vink et al. (2000) with a refined version of the semi-empirical Monte Carlo (MC) method of Abbott \& Lucy (1985). Logically, therefore, the conflict could arise from the specific assumptions of Vink et al. rather than from a failure of the radiative-driving mechanism.

In L07, MRL models were used to explore the sensitivity of the eigenvalue $J=\Phi / 4 \pi R^{2}$ to $v_{\mathrm{t}}$, the microturbulent velocity. Crucially, this parameter affects the flux irradiating lines as matter is driven through the sonic point; and an increase in $v_{\mathrm{t}}$ from 10 to $15 \mathrm{~km} \mathrm{~s}^{-1}$ was found to decrease $J$ by $\approx 0.3$ dex. Thus, a physical effect was identified that, by reducing the predicted $\Phi$ 's, might partially contribute to resolving the conflict.

More recently (Lucy 2010, L10), MRL theory was used to investigate individual stars. This was prompted by the work of Marcolino et al. (2009) on the weak wind problem, the major discrepancy for late-type $O$ dwarfs between their $\Phi_{V}$ 's and observational estimates. To investigate this, a grid of 29 models was computed from which $J$ 's for particular stars could be obtained by interpolation. The results were encouraging: although the extremely low and uncertain $J$ 's estimated by Marcolino et al. were not matched, the predicted $J$ 's were $\approx 1.4$ dex lower than the $J_{V}$ 's. Moreover, when $J$ 's were interpolated for the two strongwind $\mathrm{O} 4$ stars analysed by Bouret et al. (2005), the results were consistent with the low values found when these authors took wind clumping into account.

In the above investigation, the L07 code was deliberately not changed, thereby avoiding any suspicion that adjustments were motivated by the observational data requiring explanation. But with the technique's usefulness thus demonstrated, a possibly significant flaw is now addressed, namely the local departures from dynamical consistency that result from the simple twoparameter representation of line driving. This further development is especially appropriate since dynamical consistency in modelling transonic flow was identified in L10 as the key to accurate predictions of $J$ and $\Phi$. Accordingly, the primary purposes of this paper are first to describe how such improved models can be constructed and then to compute J's for all TLUSTY atmospheres relevant for Galactic main-sequence $\mathrm{O}$ stars.

Throughout this paper $J$ 's are in units $\mathrm{g} / \mathrm{s} / \mathrm{cm}^{2}$ and $\Phi$ 's in $\mathcal{M}_{\odot} / \mathrm{yr}$.

\section{Improved solution technique}

As in previous papers, transonic flow is assumed to be stationary, isothermal, and plane-parallel. The equation of motion can then be written as

$\left(v^{2}-a^{2}\right) \frac{1}{v} \frac{\mathrm{d} v}{\mathrm{~d} x}=-g_{\mathrm{eff}}$

here $a$ is the isothermal speed of sound, and $g_{\mathrm{eff}}=g-g_{\mathrm{e}}-g^{1}$ is the effective gravity, where $g_{\mathrm{e}}=\Gamma_{\mathrm{e}} g$ and $g^{1}$ are the radiative accelerations due to electron- and line scatterings, respectively.

For given stellar parameters, we wish to find the solution of Eq. (1) such that the flow accelerates smoothly from sub- to supersonic velocities. This is achieved by finding the particular mass flux $J$ that gives $g_{\text {eff }}=0$ at the sonic point $v=a$, thus avoiding a singularity when integrating Eq. (1).

When approximated by a MC estimator, $g^{1}(v)$ is not analytic, and so solving Eq. (1) and determining its eigenvalue $J$ is not a conventional excercise in integrating an ODE. Accordingly, in L07, a two-parameter formula was adopted for $g^{1}(v)$ that automatically gives $g_{\mathrm{eff}}=0$ at $v=a$, thus allowing the singularityfree stratification of the MRL to be obtained with a conventional 
integration of Eq. (1). The MC transfer calculation was then carried out in this stratified medium, resulting in estimates $\tilde{g}^{1}$ for each layer of the MRL. The challenge then was to find the values of $J$ and of the parameters $\delta$ and $s$ that brought $\tilde{g}^{1}$ into optimal agreement with $g^{1}(v ; J, \delta, s)$.

Because of this parametric approach, the solutions obtained had noticeable residuals $\Delta g^{1}=\tilde{g}^{1}-g^{1}-$ see Fig. 3 in L10 - implying some uncertainty in the predicted $J$ 's. Although evidence was presented that $J$ is moderately insensitive to departures from detailed local dynamical consistency, it is nevertheless desirable to eliminate this weak point in MRL theory.

\subsection{Non-parametric $g^{1}(v)$}

To allow the $\tilde{g}^{1}$ 's to be accurately modelled by $g^{1}(v)$, this function is constrained to pass through the discrete set of points $\left(g_{i}^{1}, v_{i}\right)$, with $v_{1}<v_{2}<\ldots<v_{I}$. The required continuous function $g^{1}(v)$ is then constructed as follows: by linear logarithmic interpolation between neighbouring points for $v \in\left(v_{1}, v_{I}\right)$; by setting $g^{1}(v)=g_{1}^{1}$ for $v<v_{1}$; and by extrapolating the power law from the interval $(I-1, I)$ for $v>v_{I}$.

The discrete representation extends from a small subsonic velocity $v_{1}$ to a supersonic velocity $v_{I} \geq 2 a$, with spacing chosen to model the often sharply changing velocity gradient as the sonic point is approached - see Fig. 1 in L07. The $k$ th point is located at the sonic point - i.e., $v_{k}=a-$ and the corresponding value of $g_{k}^{1}$ is constrained to be $g_{*}=g-g_{\mathrm{e}}$, so that the regularity condition is again automatically satisfied.

\subsection{Stratification}

With the $g^{1}(v)$ thus defined, the MRL's stratification is obtained as described in Sect. 2.3 of L07, namely by two initial-value integrations of Eq. (1) starting at $v=a$, one for $v<a$ and one for $v>a$.

To avoid a singularity at $v=a$, the initial velocity gradient must be such that

$\left(v \frac{\mathrm{d} v}{\mathrm{~d} x}\right)_{a}=\frac{1}{2}\left(\frac{\mathrm{d} \ln g^{1}}{\mathrm{~d} \ln v}\right)_{a} g_{*}$.

Now, if $g^{1}(v)$ were an analytic function, the logarithmic derivative in Eq. (2) would be the same for both inward and outward integrations. But the adopted piecewise-linear segmented representation of $\log g^{1}$ is not analytic: although continuous, its derivative is in general discontinuous at $v=v_{i}$ and thus may be so at $v_{k}=a$. Accordingly, for the inward and outward integrations, the logarithmic derivatives are the slopes of the $(k-1, k)$ and $(k, k+1)$ segments, respectively.

The discontinuities in the derivatives of $g^{1}(v)$ allow the representation to approximate curvature in $\left(\log g^{1}-\log v\right)$-plots. Of course, as $I \rightarrow \infty$, unlimited accuracy can be achieved, and the discontinuities then $\rightarrow 0$.

\subsection{An example}

Model $t 400 \mathrm{~g} 375$, with parameters $T_{\text {eff }}=40000 \mathrm{~K}, \log g=3.75$ and $v_{\mathrm{t}}=10 \mathrm{~km} \mathrm{~s}^{-1}$, illustrates the improved technique.

Figure 1 shows the first steps in the search for $J$. The starting values for $g_{i \neq k}^{1}$ are obtained from the two-parameter formula see Eq. (1) in L10 - with $\delta=0.5$ and $s=1.5$ - i.e., a broken power law, with the switch to a rapidly increasing $g^{1}(v)$ occurring at Mach number $m=v / a=0.63$. With $g^{1}(v)$ thus fixed, several models were computed with varying $J$ in order to locate the root

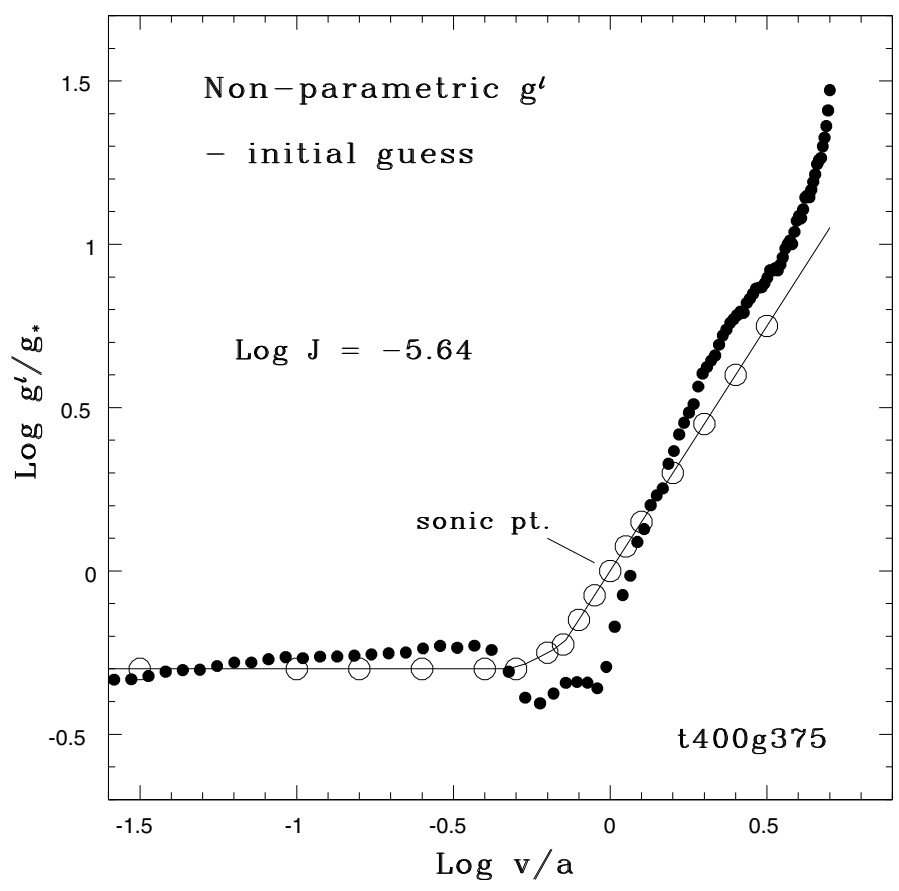

Fig. 1. Search for dynamical consistency. The open circles are the initial discrete representation $\left(g_{i}^{1}, v_{i}\right)$ for model $t 400 g 375$, and the connecting solid line is the resulting continuous function $g^{\mathrm{l}}(v)$. The MC estimates $\tilde{g}^{1}$ are plotted as filled circles and correspond to the mass flux $J=-5.64$ dex that gives $Q_{1,2}=0$.

of $Q_{1,2}(J)=0-$ see Sect. 2.3 and Fig. 2 in L10. The result, $J=-5.64 \mathrm{dex}$, is then such that, as matter accelerates from $m_{1}=0.5$ to $m_{2}=2.0$, the work done by the gradients of gas and radiation pressures accounts for the gain in mechanical energy per $g$. Nevertheless, the non-vanishing residuals $\Delta g^{1}$ imply that the $I-1$ values $g_{i \neq k}^{1}$ require adjustment.

Ideally, corrections to $g_{i \neq k}^{1}$ should be derived from the $\Delta g^{1}$ with an algorithm analogous to the temperature-correction procedures in stellar atmosphere theory. But here a trial-and-error procedure is followed based on inspection of plots such as Fig. 1. Thus, Fig. 1 shows that a steeper slope than $s=1.5$ is required for $m>1$ and that the $g_{i}^{1}$ should be increased by $\approx 0.1$ dex for $m \in(0.1,0.4)$.

With the $g_{i \neq k}^{1}$ thus adjusted, a new sequence of models is computed, the modified root of $Q_{1,2}=0$ derived, and an updated version of Fig. 1 plotted. This iterative procedure is continued until a satisfactory degree of convergence is achieved. In this case, the final model has $J=-5.72$ dex and is plotted in Fig. 2 . The iteratively-corrected function $g^{1}(v)$ now agrees closely with the $\tilde{g}^{1}$ 's. A dynamically consistent model of turbulent transonic flow has therefore been constructed.

\section{Microturbulence}

As demonstrated in L07, the $J$ 's predicted by MRL theory are sensitive to $v_{\mathrm{t}}$, which must therefore be included with $T_{\text {eff }}$ and $g$ when comparing with observational data.

Given the importance of this sensitivity, the improved code is now applied to the model $t 400 \mathrm{~g} 375$ in order to check and extend the analysis of L07. As in that investigation, when $v_{\mathrm{t}}$ is varied, the b-values and incident flux distribution are from the TLUSTY model with $v_{\mathrm{t}}=10 \mathrm{~km} \mathrm{~s}^{-1}$.

Solutions ranging from $v_{\mathrm{t}}=0$-i.e., pure thermal broadening in the lines' Doppler cores - to $v_{\mathrm{t}}=20 \mathrm{~km} \mathrm{~s}^{-1}$, corresponding to 


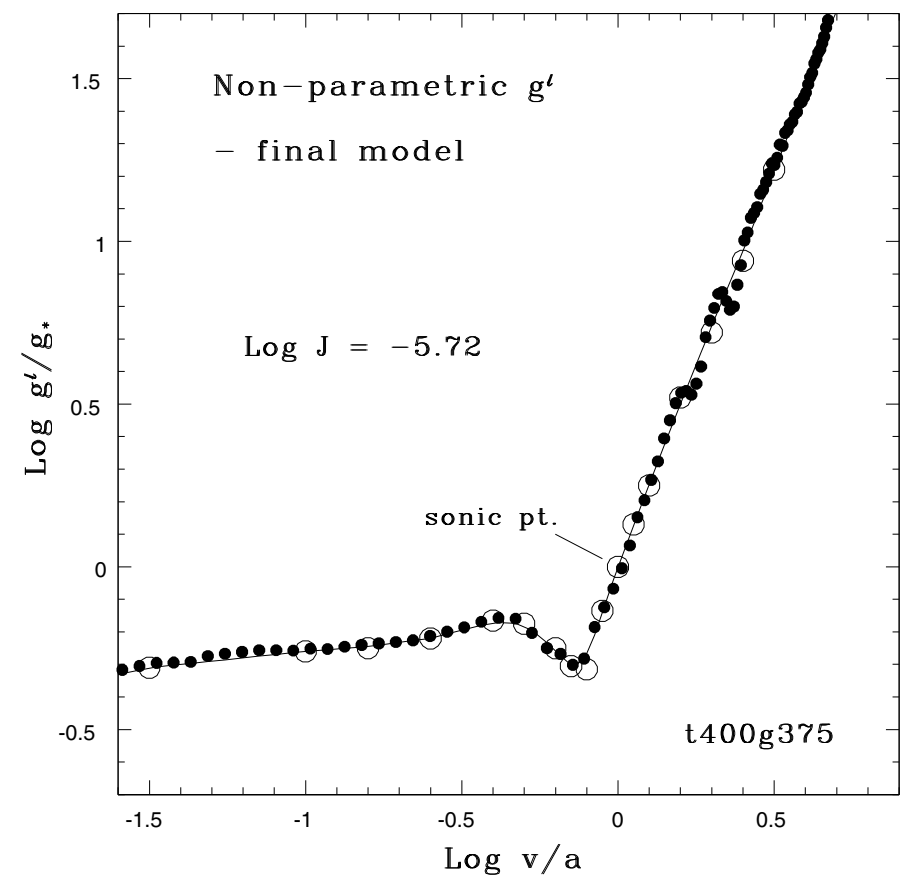

Fig. 2. Search for dynamical consistency. Symbols and model parameters as in Fig. 1. The interatively-corrected representation $\left(g_{i}^{1}, v_{i}\right)$ is shown together with the resulting MC estimates $\tilde{g}^{\mathrm{l}}$ when $J=-5.72$ dex.

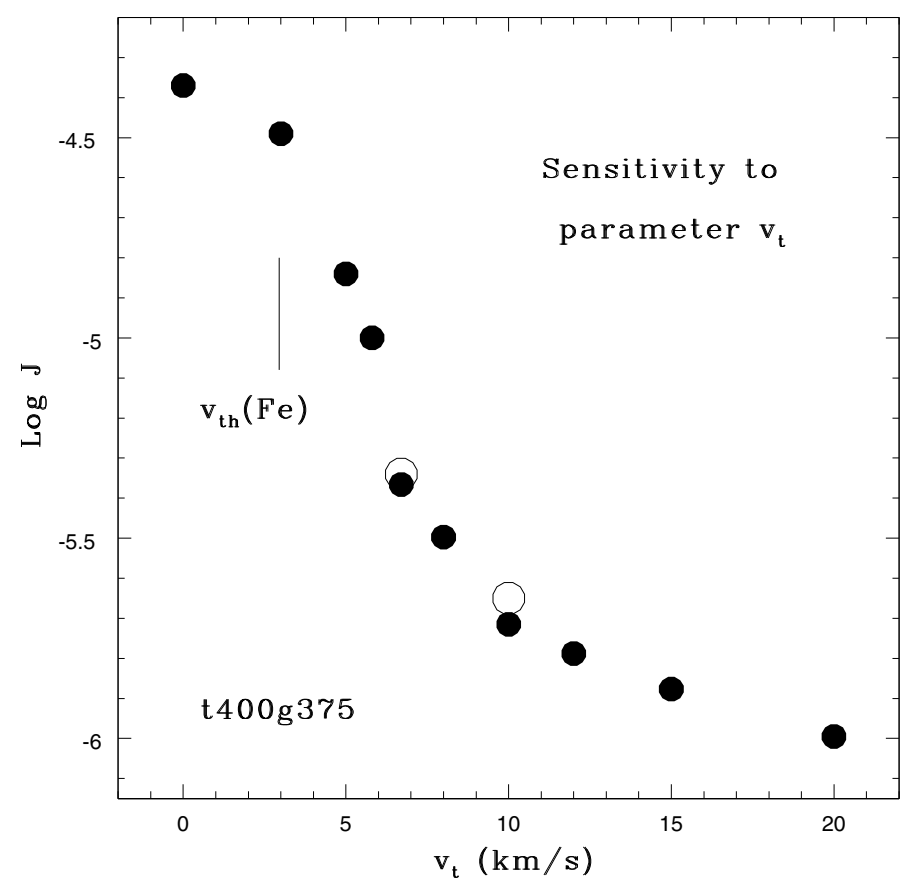

Fig. 3. Sensitivity of the eigenvalue $J$ to microturbulent velocity $v_{\mathrm{t}}$ for model $t 400 g 375$. Filled circles are solutions obtained as described in Sect. 2; open circles are solutions from L07. The thermal speed of $\mathrm{Fe}$ ions is indicated.

near sonic turbulence, are plotted in Fig. 3, together with the two solutions from L07 at 6.7 and $10 \mathrm{~km} \mathrm{~s}^{-1}$. The sensitivity to $v_{\mathrm{t}}$ is confirmed.

From the new results between 6.7 and $15 \mathrm{~km} \mathrm{~s}^{-1}$, the logarithmic slope at $10 \mathrm{~km} \mathrm{~s}^{-1}$ is

$\frac{\partial \log J}{\partial \log v_{\mathrm{t}}}=-1.46$

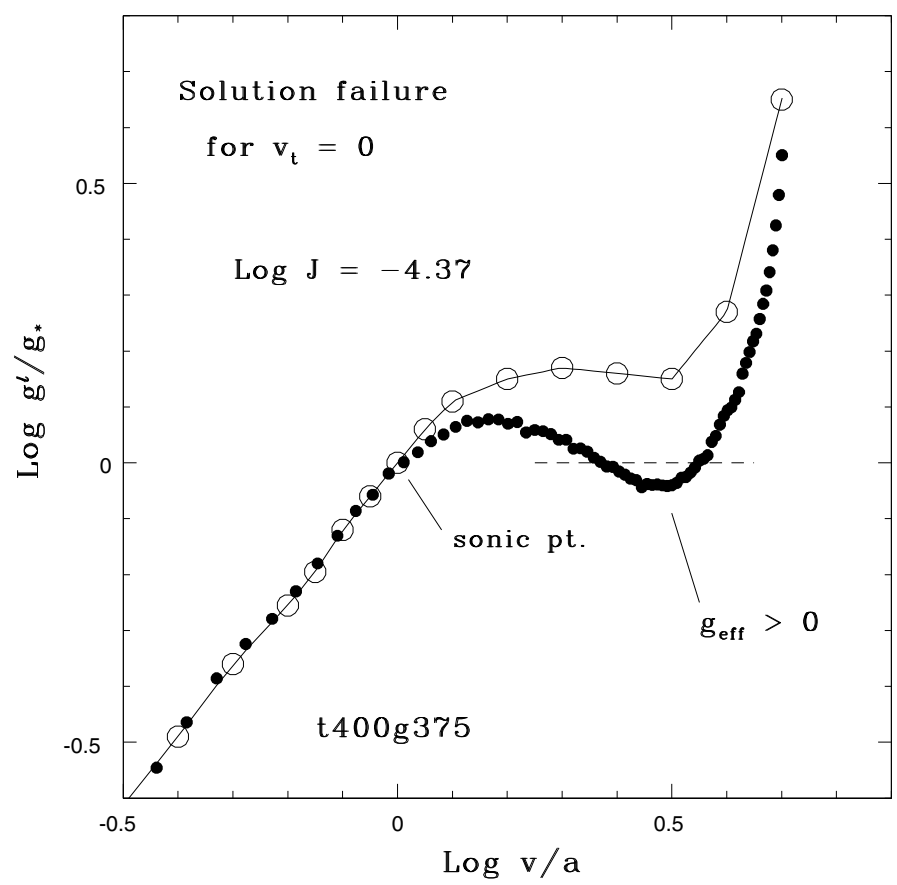

Fig. 4. Failed search for dynamical consistency when $v_{\mathrm{t}}=0$ for model t400g375. Symbols as in Fig. 1.

This value is recommended for propagation-of-error calculations of $\sigma_{\log J}$ for stars with $v_{\mathrm{t}}$ 's comparable to the canonical $v_{\mathrm{t}}=10 \mathrm{~km} \mathrm{~s}^{-1}$.

Figure 3 shows that, in principle, the parameter $v_{\mathrm{t}}$ could bring about differences in $J$ by $\gtrsim 1$ dex at fixed $T_{\text {eff }}, g$. But as this seems not to happen for real stars, the mechanism exciting and maintaining microturbulence is presumably preventing the independent variation of this parameter, resulting perhaps in a functional dependence of $v_{\mathrm{t}}$ on $T_{\mathrm{eff}}, g$.

In view of the strong damping expected for turbulence with $v_{\mathrm{t}} / a \sim 0.5$, work done by radiative forces is almost certainly required for its maintenance, in which case the phenomenon of microturbulence is not unrelated to radiatively-driven outflows. In fact, Fig. 3 suggests a direct, causal relationship as follows: given the observational evidence that wind-clumping occurs shortly after the sonic point (Bouret et al. 2005), some clumps may well lose their net outward driving and thus fall back into the photosphere (e.g., Howk et al. 2000) where their dissipated kinetic energy could excite and maintain local turbulence. If the fall-back fraction decreases with decreasing $J$, a feedback loop operates, so that for a given star only one pairing $\left(J, v_{\mathrm{t}}\right)$ is possible.

This conjectured coupling of $J$ and $v_{\mathrm{t}}$ is supported by the difficulty of obtaining satisfactory high mass flux MRL's when $v_{\mathrm{t}} \rightarrow 0$. The $J$ 's for $v_{\mathrm{t}} \lesssim 5 \mathrm{~km} \mathrm{~s}^{-1}$ in Fig. 3 are estimated by achieving dynamical consistency only for $v / a<1$ since the extension to $v / a \gtrsim 2$ is not possible because $g_{\text {eff }}$ becomes positive see Fig. 4. Admittedly, this breakdown of the iterative procedure is code-specific, resulting from choosing $v$ rather than height $x$ as the independent variable. Nevertheless, even if the code were reconfigured to allow dynamical consistency to be extended to supersonic velocities, the resulting non-monotonic velocity law implies a density inversion at $v / a \sim 2-3$. Such a stratification is surely unstable and thus a possible origin of clumping and of infalling blobs. 
Table 1. Computed mass fluxes $J$.

\begin{tabular}{ccccc}
\hline \hline $\log g$ & $T_{\text {eff }}(\mathrm{kK})$ & $\log J$ & $T_{\text {eff }}(\mathrm{kK})$ & $\log J$ \\
\hline 4.50 & 27.5 & -7.78 & 42.5 & -6.05 \\
& 30.0 & -8.67 & 45.0 & -5.87 \\
& 32.5 & -8.02 & 47.5 & -5.67 \\
& 35.0 & -7.45 & 50.0 & -5.40 \\
& 37.5 & -6.84 & 52.5 & -5.11 \\
& 40.0 & -6.34 & 55.0 & -4.87 \\
\hline 4.25 & 27.5 & -7.58 & 42.5 & -5.95 \\
& 30.0 & -8.04 & 45.0 & -5.84 \\
& 32.5 & -7.56 & 47.5 & -5.52 \\
& 35.0 & -7.22 & 50.0 & -5.28 \\
& 37.5 & -6.62 & 52.5 & -4.74 \\
& 40.0 & -6.26 & 55.0 & -4.48 \\
\hline 4.00 & 27.5 & -7.45 & 42.5 & -5.70 \\
& 30.0 & -7.54 & 45.0 & -5.54 \\
& 32.5 & -7.18 & 47.5 & -4.98 \\
& 35.0 & -6.88 & 50.0 & -4.46 \\
& 37.5 & -6.44 & 52.5 & -4.18 \\
& 40.0 & -5.99 & 55.0 & -3.79 \\
\hline 3.75 & 27.5 & -7.72 & 40.0 & -5.72 \\
& 30.0 & -7.40 & 42.5 & -5.14 \\
& 32.5 & -7.11 & 45.0 & -4.86 \\
& 35.0 & -6.86 & 47.5 & -4.31 \\
& 37.5 & -6.04 & & \\
\hline 3.50 & 27.5 & -7.72 & 35.0 & -6.10 \\
& 30.0 & -7.07 & 37.5 & -4.85 \\
& 32.5 & -6.33 & 40.0 & -4.42 \\
\hline 3.25 & 27.5 & -7.40 & 32.5 & -5.47 \\
& 30.0 & -6.51 & 35.0 & -4.79 \\
\hline 3.00 & 27.5 & -6.95 & 30.0 & -5.40 \\
\hline & & & & \\
& & &
\end{tabular}

\subsection{A spectroscopic test}

This sensitivity of $J$ to $v_{\mathrm{t}}$ is in stark contrast to CAK theory (Castor et al. 1975), which posits that the properties of radiatively-driven winds can be derived on the basis of the Sobolev approximation. In this approximation, $g^{1}(v)$ is independent of the lines' absorption profiles and therefore independent of $v_{\mathrm{t}}$ 's contribution to the width of the Doppler core.

In principle, this can be tested observationally, and is best done differentially. Thus stars differing in $v_{\mathrm{t}}$ but not widely separated in $\left(T_{\text {eff }}, g\right)$-space should be subjected to identical observing and diagnostic procedures to see if $\Delta \Phi$ can be understood without (CAK), or only with (MRL), a contribition from $\Delta v_{\mathrm{t}}$.

\section{Computed mass fluxes}

In this section, the improved technique of Sect. 2 is used to recalculate the 29 models in Table 1 of L10 and then to add a further 28 models in order to provide a rather complete coverage of $\left(T_{\mathrm{eff}}, g\right)$-space for $\mathrm{H}$-burning $\mathrm{O}$ stars. As before, the models' composition is solar with $N_{\mathrm{He}} / N_{\mathrm{H}}=0.1$ (Grevess \& Sauval 1998), the included metal ions are as in Table 1 of Lanz $\&$ Hubeny (2003), and $v_{\mathrm{t}}=10 \mathrm{~km} \mathrm{~s}^{-1}$.

\subsection{O-star grid}

Mass fluxes $J$ for 57 models are given in Table 1. The grid is determined by the availability of TLUSTY atmospheres (Lanz \& Hubeny 2003) and is complete for their models with $\log g \leq 4.5$. Because $\mathrm{O}$ stars on the ZAMS have $\log g \approx 4.2-$ see Fig. 4 in L10, $J$ 's have not been computed for $\log g=4.75$.

The coverage provided by Table 1 allows $J$ 's to be determined for all Galactic O stars by interpolation - or a slight

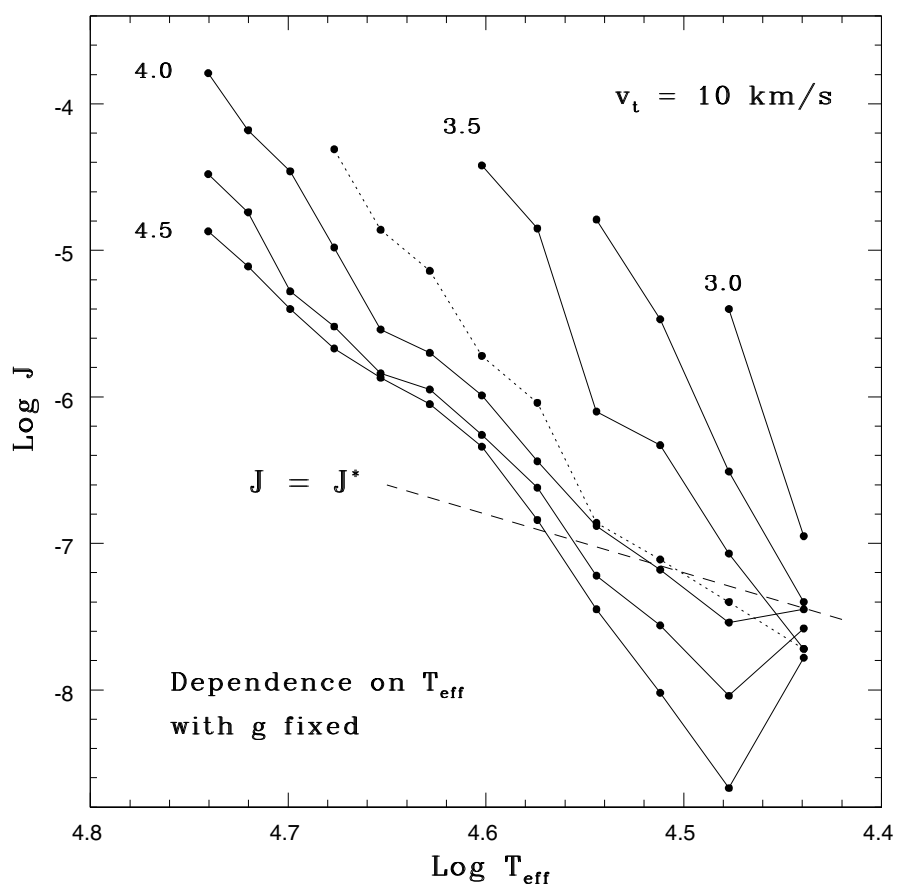

Fig. 5. Mass flux $J$ as a function of $T_{\text {eff }}$ for $\log g=3.00(0.25) 4.50$. The data are from Table 1 . The dashed line $J=J^{*}$ defines the boundary of the weak-wind domain - see L10.

extrapolation in a few cases. If, in addition to $T_{\text {eff }}, g$ and $v_{\mathrm{t}}$, a star's distance is known, its radius can be computed and therefore also $\Phi=4 \pi R^{2} J$. Given the detailed diagnostic modelling of numerous $\mathrm{O}$ stars in recent years, a critical evaluation of MRL theory may be possible with existing data. This is not attempted here and is, in any case, best carried out by investigators familiar with the uncertainties of analysing circumstellar spectra.

The data in Table 1 is also relevant for investigations of stellar evolution with mass loss, for computing the latitude dependence of mass loss for rapidly-rotating stars, and for calculating its radial dependence for accretion disks.

The dependence of $J\left(T_{\text {eff }}, g ; v_{\mathrm{t}}\right)$ on $T_{\text {eff }}$ and $g$ is shown in Figs. 5 and 6. The most striking feature is the departure from the expected monotonic increase of $J$ with increasing $T_{\text {eff }}$ that occurs when $T_{\text {eff }} \lessgtr 30000 \mathrm{~K}$ and $\log g \gtrsim 3.9$. As discussed in L10, this prediction of MRL theory offers at least a partial explanation of the weak-wind phenomenon. Interestingly, the minimum at $T_{\mathrm{eff}} \approx 30000 \mathrm{~K}$ is deeper for $g>g_{\mathrm{ZAMS}}$.

\subsection{Accuracy}

The J's in Table 1 have several sources of uncertainty. The first originates from MC sampling errors - see Appendix A. The $\tilde{g}^{1}$ s sampling errors propagate via $Q_{1,2}$ into errors in $J$ when this quantity is determined by locating the intercept $Q_{1,2}(J)=0$ - see Fig. 2 in L10. Thus, for the final $t 400 \mathrm{~g} 375$ model in Fig. 2, the least squares solution is $\log J=-5.715 \pm 0.014$. This estimate of $\sigma_{\log J}$ shows that MC noise is inconsequential since other errors are surely far greater.

The uncertainty $\sigma_{\log J} \rightarrow 0$ as the number of MC quanta $N \rightarrow \infty$, but $J$ would still be subject to error because of residual departures from exact dynamical consistency if the continuous function $g^{1}(v)$ derives from only a finite number of points $\left(g_{i}^{1}, v_{i}\right)$.

This second source of uncertainty can be estimated as follows: given that the non-parametric representation is a marked improvement over the previous model, the average $|\Delta \log J|$ 


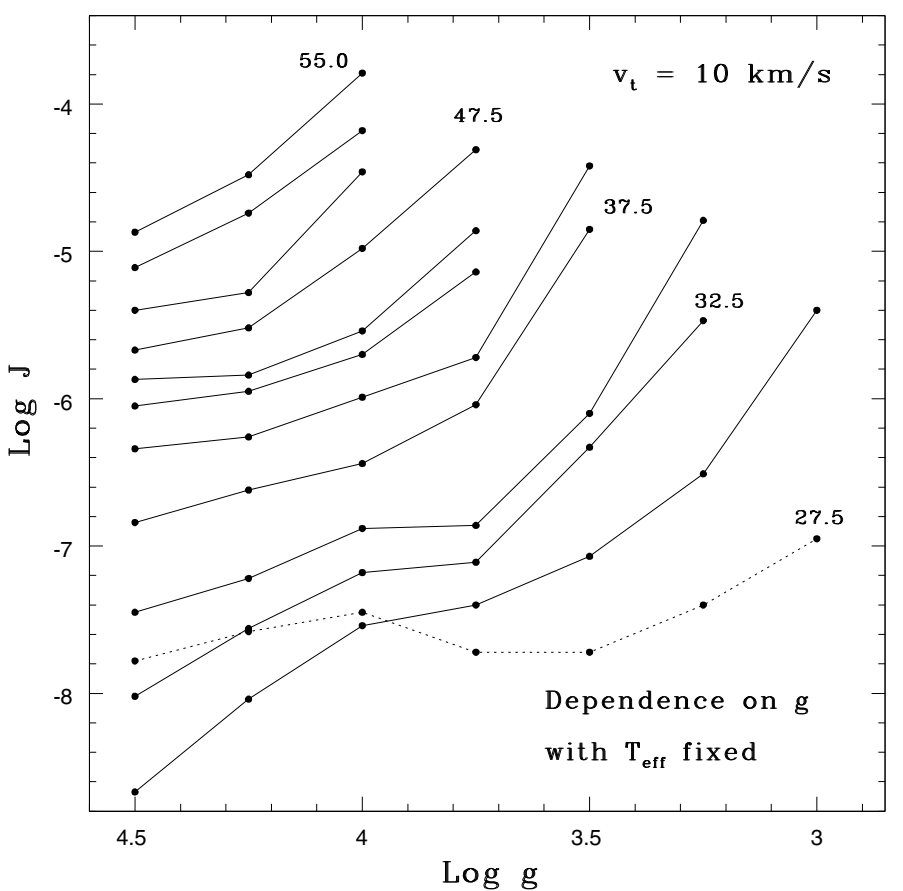

Fig. 6. Mass flux $J$ as a function of $g$ for $T_{\text {eff }}=27.5(2.5) 55.0 \mathrm{kK}$. The data are from Table 1.

between the models treated both here and in L10 is a good estimate of the typical error of the previous $J$ 's and, at the same time, a conservative error estimate for the $J$ 's in Table 1 . The $29 \mathrm{~J}$ 's differ on average by only $0.10 \mathrm{dex}$, with the largest difference being 0.33 dex.

A third source of uncertainty is the cumulative effect of errors in abundances, input physics and line-formation theory, many of which propagate from the TLUSTY models. A plausible guess is that these errors should rarely exceed 0.2 dex.

A fourth source of uncertainty is the derivation of $J$ from a plane-parallel treatment of transonic flow, with back-scattering from $v>5 a$ neglected. This is investigated in Appendix B and found to be well-justified.

A fifth and probably dominant source of uncertainty is the sensitivity to the throttling effect of turbulent line broadening (L07 and Sect. 3 above). Given our ignorance as to the source and nature of photospheric turbulence, this has perforce been investigated in the microturbulent limit, and strong sensitivity is found. Thus, from Fig. 3, we see that, with $v_{\mathrm{t}}=10 \mathrm{~km} \mathrm{~s}^{-1}$, photospheric turbulence reduces $J$ by $\approx 1.3 \mathrm{dex}$ from its value for laminar flow - i.e., pure thermal broadening. Within the context of the microturbulent model, an error of $\pm 2 \mathrm{~km} \mathrm{~s}^{-1}$ at $v_{\mathrm{t}} \approx 10 \mathrm{~km} \mathrm{~s}^{-1}$ implies $\sigma_{\log J} \approx 0.13$ according to Eq. (3).

Given these uncertainties, tests of MRL theory, either spectroscopically or via stellar evolution calculations, might reasonably allow for an uncertainty of $\pm 0.2 \mathrm{dex}$ in the $J$ 's given in Table 1 . But if a test reveals systematic discrepancies $>0.4 \mathrm{dex}$, a contradiction can be claimed.

\section{Comparisons with mass-loss formulae}

In this section, the MRL mass fluxes are compared to widelyused mass-loss formulae. The aim here is not a comprehensive discussion but to call attention to opportunities for differential testing, as already discussed in Sect. 3.1.

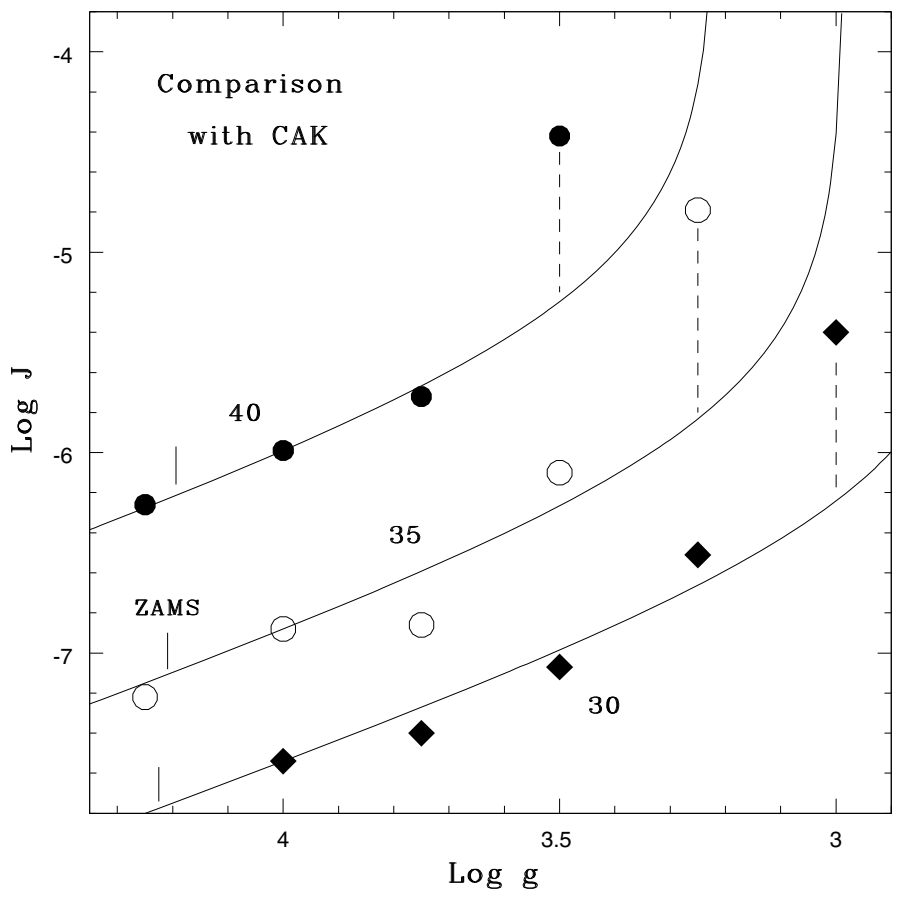

Fig. 7. Comparison of MRL mass fluxes $J_{L}$ with scaled CAK formulae for $T_{\text {eff }}=30,35$ and $40 \mathrm{kK}$. The dashed vertical lines are the MRLCAK offsets discussed in the text.

\subsection{CAK}

In their recent discussion of WNH stars, Smith \& Conti (2008) sketch an evolutionary scenario based on an O-star mass-loss formula extracted from CAK theory. For fixed $T_{\text {eff }}$, their formula gives $J$ 's dependence on $g=g_{\mathrm{e}} / \Gamma_{\mathrm{e}}$ as

$J=J_{0} \frac{\Gamma_{\mathrm{e}}}{1-\Gamma_{\mathrm{e}}}$

where $J_{0}$ is the mass flux when $\Gamma_{\mathrm{e}}=0.5$. Equation (4) predicts that $J \rightarrow \infty$ as $\Gamma_{\mathrm{e}} \rightarrow 1-$ i.e., as the Eddington limit is approached - and this is basic to their claim that a feedback process results in runaway mass loss late in the core-H burning phase of massive $\mathrm{O}$ stars.

In Fig. 7, the behaviour of $J_{L}$ as $g \rightarrow g_{\mathrm{e}}$ is compared to Eq. (4) when $J_{0}$ is chosen to match $J_{L}$ at $\log g=4$. This comparison is carried out for $T_{\text {eff }}=30,35$ and $40 \mathrm{kK}$, for which the lowest $g$ TLUSTY models have $\Gamma_{\mathrm{e}}=0.52,0.54$ and 0.52 , respectively. Remarkably, MRL theory predicts greatly enhanced mass loss when a star is still well-detatched from its Eddington limit. In each of the plotted sequences, the lowest $g$ model is offset from the scaled CAK formula by $\Delta J \sim 1 \mathrm{dex}$.

If the Smith-Conti scenario were supported by actual stellar evolution tracks and accurately fitted several well-observed WNH stars, then MRL theory could be immediately dismissed as over-predicting $J$ for evolved $\mathrm{O}$ stars. But neither of these circumstances obtains, and this enhanced mass loss already at $\Gamma_{\mathrm{e}} \approx 0.5$ will likely prove favourable to their scenario. Clearly, further work is required on the evolution of mass-losing massive stars.

In addition to its relevance for evolutionary scenarios, Fig. 7 suggests another differential test to distinguish MRL and CAK theories. In this case, pairs of stars with closely similar $T_{\text {eff }}$ 's but markedly different $g$ 's should be observed and analysed identically to see which $g$-dependence in Fig. 7 is favoured. 


\subsection{Vink et al.}

A model from Table 1 cannot be directly compared to the Vink et al. formula since the latter requires three fundamental stellar parameters and $v_{\infty}$. Accordingly, the comparison is carried out for the ZAMS models of Pols et al. (1998) with $Z=0.02$. Each point on the ZAMS gives $\mathcal{M}, R$ and $L$ which, with the additional assumption that $v_{\infty} / v_{\text {esc }}=2.6$ (Lamers et al. 1995), allows $\Phi_{V}$ to be computed from Eq. (12) of Vink et al. (2000). This can then be compared to $\Phi_{L}=4 \pi R^{2} \times J\left(T_{\text {eff }}, g\right)$. Here $J$ is obtained by simple bivariate interpolation (Abramowitz \& Stegun 1965) from the four surrounding entries in Table 1, taking the independent variables to be $\log T_{\text {eff }}$ and $\log g$.

The two predictions for the ZAMS are plotted in Fig. 8. Throughout the entire range $\Delta \Phi=\Phi_{V}-\Phi_{L}>0$. At $T_{\text {eff }}=$ $27650 \mathrm{~K}$, the offset is $0.40 \mathrm{dex}$, and this increases steeply to a maximum of 1.24 dex at $30150 \mathrm{~K}$. Thereafter, $\Delta \Phi$ decreases not quite monotonically - to reach a barely significant 0.27 dex at $T_{\text {eff }}=50300 \mathrm{~K}$.

The huge difference at $T_{\text {eff }} \approx 30000 \mathrm{~K}$ allows MRL theory to partly explain the weak wind phenomenon, which arose when the Vink et al. predictions were compared to mass loss estimates for late-type $\mathrm{O}$ dwarfs. In fact, the relevant diagnostic analyses (Marcolino et al. 2009) appear still to require $\Phi_{L}$ to be reduced by $\approx 0.8$ dex - see L10.

According to Fig. 8, MRL theory predicts that a massive star's initial $\Phi$ is markedly less than $\Phi_{V}$. However, when its expanding radius has reduced $g$ by $\approx 1$ dex, the situation reverses see Fig. 7. Thus, for example, if a mass-losing star reaches the point $(40000 \mathrm{~K}, 3.5)$ in $\left(T_{\text {eff }}, \log g\right)$-space with $\mathcal{M} / \mathcal{M}_{\odot}=80$, then $\Phi_{V}=-4.75 \mathrm{dex}$, but $\Phi_{L}=-4.59 \mathrm{dex}$, a factor 1.4 larger. To put this in context, the $120 \mathcal{M}_{\odot}$ track of Limongi \& Chieffi (2006) computed with Vink et al. mass loss has log $g=3.56$ and 3.31 with mass 93.6 and $56.6 \mathcal{M}_{\odot}$, respectively, at its two crossings of $T_{\text {eff }}=40000 \mathrm{~K}$ during core H-burning.

From the trends evident in Fig. 7, the MRL mass-loss enhancement will be even greater for $g$ 's smaller than given in Table 1. Moreover, the Limongi-Chieffi track suggests that this part of parameter space may well be accessed by real stars. Accordingly, Table 1 needs to be extended to lower $g$ 's, and this will require an improved theory - see Appendix B.

\section{Conclusion}

Motivated by the (partial) successes of MRL theory in reproducing the reduced $\Phi$ 's found by spectroscopists for O stars when wind-clumping is taken into account, the aim of this paper has been to complete the coverage of the relevant $\left(T_{\text {eff }}, g\right)$-domain provided by the TLUSTY atmospheres. To this end, the MRL code has been improved by adopting a non-parametric description of $g^{1}(v)$, which has allowed a greater degree of dynamical consistency to be achieved for turbulent transonic flow, and thus more accurate $J$ 's. The results of this effort are the 57 values of $\log J$ in Table 1.

Interpolation in Table 1 allows $J$ to be derived for any $\mathrm{O}$ star with measured $T_{\text {eff }}$ and $g$. Moreover, a propagation-of-error calculation gives $\sigma_{\log J}$ if the standard errors of these two parameters and of $v_{\mathrm{t}}$ have been determined. This can be done for stars individually and independently. However, given the difficulties of diagnostic analyses, with the resulting possibility of systematic errors, there is merit in performing differential tests as suggested in Sects. 3.1 and 5.1. If $v_{\mathrm{t}}$ is a slowly-varying function of $T_{\text {eff }}$ and $g$, the first test may be indecisive. But the proposed test between the MRL and CAK theories suggested by Fig. 7 is

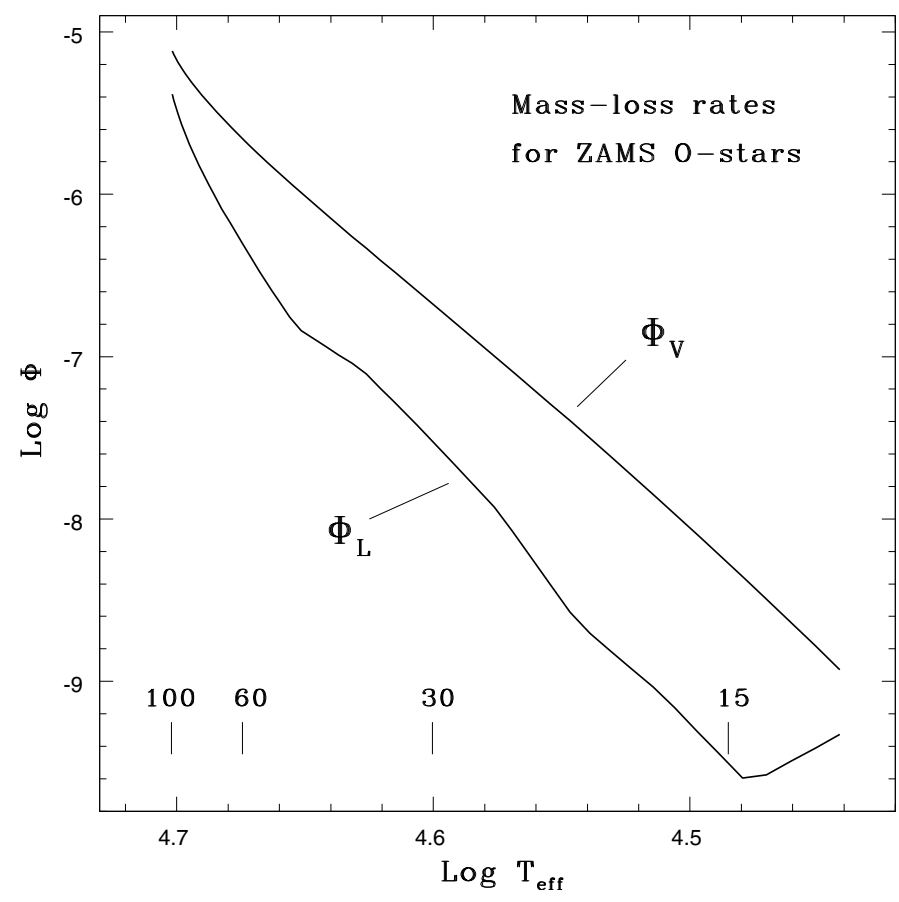

Fig. 8. Mass-loss rates for O-stars on the zero-age main sequence (ZAMS) as a function of $T_{\text {eff }}$, with masses in solar units indicated. Predictions of MRL theory $\left(\Phi_{L}\right)$ are plotted as well as values $\left(\Phi_{V}\right)$ derived with the formula of Vink et al. (2000).

feasible. Moreover, this test is fundamental for stellar wind theory since it directly concerns the question: where in the outflow is the mass-loss rate determined?

With regard to this question, the partial success of MRL theory in explaining the weak wind phenomenon supports the LS70 argument that $J$ and therefore $\Phi$ is determined by the regularity condition at the sonic point. This would be decisive if the observed $\Phi^{\prime} s$ of the Marcolino et al. (2009) stars were convincingly revised into agreement with the predictions of MRL theory. But since only weak C IV absorption is observed, there is little or no observational basis for such improved estimates. Other tests should therefore be carried out.

Acknowledgements. I am grateful to M. Limongi and A. Chieffi for unpublished details of their evolutionary tracks and to the referee, A. de Koter, for his thoughtful comments on the proposed differential tests.

\section{Appendix A: Precision of the estimator $\tilde{\boldsymbol{g}}^{\mathrm{I}}$}

The $\tilde{g}^{1}$ are derived from a MC simulation using estimator $A$, the summation over pathlengths given in Eq. (10) of L07. This is expected to be more accurate than estimator $B$, the summation of momentum tansfers from energy packets to matter at the discrete line-scattering events.

A test of the accuracy and convergence of $A$ and $B$ has been carried out for model $t 500 \mathrm{~g} 400$. In this test, the exact $g^{1}$ is taken to be $\tilde{g}^{1}$ given by $A$ when $N$, the number of MC quanta, is $512 \times 10^{6}$. Given this "exact" $g^{1}$, the fractional errors of $\tilde{g}^{1}$ can be computed at smaller $N$ for $A$ and at all $N$ for $B$.

The mean absolute fractional errors for $0.5<v / a<2$ are plotted against $N$ in Fig. A.1. As expected, errors for both $A$ and $B$ are $\propto 1 / \sqrt{N}$. Also as expected, $A$ is the more accurate. From the plotted least squares fits, $\epsilon_{A}=0.488 \times \epsilon_{B}$. Thus, to achieve the same accuracy, $B$ would have required $N$ to be increased by 


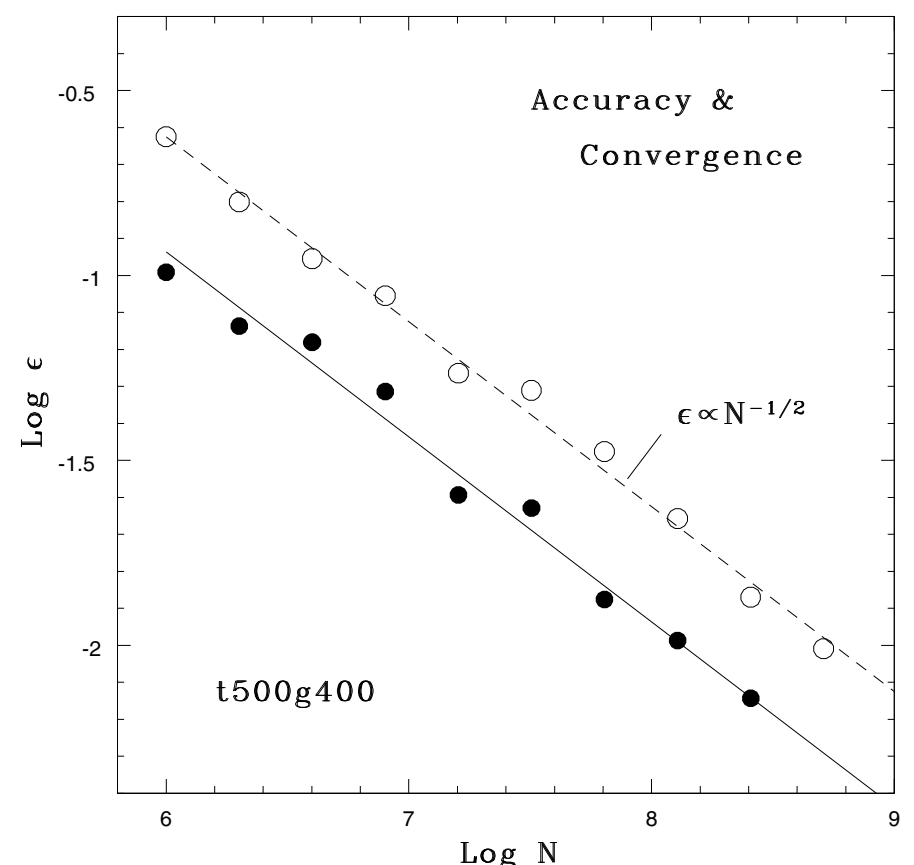

Fig. A.1. Convergence and accuracy test of MC estimators. For model $t 500 \mathrm{~g} 400$, the mean absolute fractional error $\epsilon$ of $\tilde{g}^{1}$ for $0.5<v / a<2$ is plotted against $N$, the number of energy packets. The filled and open circles refer to estimators $A$ and $B$, repectively.

the factor 4.2. The saving of computer time with $A$ was essential in carrying out the huge modelling effort required to produce Table 1.

A typical simulation has $N=40 \times 10^{6}$ and so, according to Fig. A.1, the typical error of $\tilde{g}^{1}$ with $A$ is 0.016 dex. But this is specific to model $t 500 \mathrm{~g} 400$. At cooler $T_{\text {eff' }}$ 's, an increasing fraction of packets propagate through the MRL without undergoing line scatterings, so that, for fixed $N$, the sampling error of $\tilde{g}^{1}$ increases. Thus, for model $t 325 \mathrm{~g} 400$, the above error increases to 0.20 dex.

\section{Appendix B: Reflection probability}

When a MC quantum crosses the upper boundary of the MRL at height $x_{1}$ where $v / a=5$, it permanently exits the computational domain. Thus, its reflection probability $p_{1}=0$, an assumption justified if the true $p_{1} \ll 1$.

An estimate of $p_{1}$ can be derived following the method of Abbott \& Lucy (1985, Sect. III). We assume that radiative driving dominates other mechanisms beyond $x_{1}$ and accelerates the wind to terminal velocity $v_{\infty}$. On this assumption, increases in the mechanical luminosity

$$
\mathcal{L}(r)=\Phi\left(\frac{1}{2} v^{2}-\frac{G \mathcal{M}}{r}\right)
$$

are accounted for by a matching decrease in the radiative luminosity $L(r)$. Thus, in the spherical shell $(r, r+\mathrm{d} r)$, the $O(v / c)$ difference between the rates at which matter absorbs $d \mathcal{A}$ and emits $\mathrm{d} \mathcal{E}$ radiant energy is

$$
\mathrm{d} \mathcal{A}-\mathrm{d} \mathcal{E}=\mathrm{d} \mathcal{L}=\Phi\left(v \frac{\mathrm{d} v}{\mathrm{~d} r}+g\right) \mathrm{d} r
$$

If we now assume that the electron- and line-scatterings responsible for the energy transfer absorb from a radially-streaming radiation field and emit isotropically, then

$\mathrm{d} \mathcal{E}=\frac{c}{v} \mathrm{~d} \mathcal{L}$.

Since this energy is radiated isotropically, the fraction propagating back through $r=r_{1}$ - i.e., back into the MRL - is

$w_{1}=\frac{1}{2}\left[1-\sqrt{1-\left(z / z_{1}\right)^{2}}\right]$,

provided that no interactions intervene. Here $z=R / r$, where $R$ is the photospheric radius.

Combining the above, we find that the luminosity of inwardly-propagating radiation at $r_{1}$ is

$L_{1}^{-}=\int_{r_{1}}^{\infty} w_{1} \frac{c}{v} \frac{\mathrm{d} \mathcal{L}}{d r} \mathrm{~d} r$.

The reflection probability is then $p_{1}=L_{1}^{-} /\left(L_{*}+L_{1}^{-}\right)$, where $L_{*}$ is the luminosity the wind-free star.

In evaluating $p_{1}$, we assume $L_{1}^{-} \ll L_{*}$ and that the supersonic wind obeys a $\beta$-velocity law with $\beta=1$. The result is

$p_{1}=\frac{\Phi}{\Phi_{\dagger}} \int_{0}^{z_{1}} w_{1}\left[1+\frac{\eta}{1-z}\right] \mathrm{d} z$

here $\Phi_{\dagger}=L_{*} / c v_{\infty}\left(\right.$ Cassinelli \& Castor 1973) and $\eta=\left(v_{\text {esc }} / v_{\infty}\right)^{2}$, where $v_{\text {esc }}$ is the escape velocity from $r=R$.

Values of $p_{1}$ have been computed for the ZAMS models in Fig. 8. Thus, at $\mathcal{M}=30 \mathcal{M}_{\odot}$ with $v_{\infty} / v_{\text {esc }}=2.6$, the ratio $\Phi_{L} / \Phi_{\dagger}=0.044$. Then, for $v_{1}=5 a, z_{1}=0.969$, and we find $p_{1}=0.0071$. The neglect of back-scattering from the exterior wind is therefore justified. Moreover, since $1-z_{1} \ll 1$, the assumption of plane-parallel geometry is also justified.

But for very massive ZAMS stars, the assumption $p_{1}=0$ is less valid. Thus, for $\mathcal{M}=80 \mathcal{M}_{\odot}, \Phi_{L} / \Phi_{\dagger}=0.384, z_{1}=0.972$, and we find that $p_{1}=0.064$. Accordingly, when theory and observation agree to $\lesssim 0.1 \mathrm{dex}$, further progress will require an improved treatment of transonic flow.

Similarly, for evolved stars, the enhanced mass loss when $\Gamma_{\mathrm{e}} \approx 0.5$ - see Sect. 5 - also brings about an incipient failure of the MRL model. Thus, a star with $\mathcal{M} / \mathcal{M}_{\odot}=80$ at $(40000 \mathrm{~K}, 3.5)$ in $\left(T_{\text {eff }}, \log g\right)$-space has $\Phi_{L} / \Phi_{\dagger}=2.20$ and $z_{1}=0.964$, so that $p_{1}=0.348$. Thus the radiative coupling of the MRL to the exterior wind ceases to be negligible.

\section{References}

Abbott, D. C., \& Lucy, L. B. 1985, ApJ, 288, 679

Abramowitz, M., \& Stegun, I. A. 1965, in Handbook of Mathematical Functions (New York: Dover), 882

Bouret, J.-C., Lanz, T., \& Hillier, D. J. 2005, A\&A, 438, 301

Cassinelli, J. P., \& Castor, J. I. 1973, ApJ, 179, 189

Castor, J. I., Abbott, D. C., \& Klein 1975, ApJ, 195, 157 (CAK)

Fullerton, A. W., Massa, D. L., \& Prinja, R. K. 2006, ApJ, 637, 1025

Grevess, N., \& Sauval, A. J. 1998, Sp. Sci. Rev., 85, 161

Howk, J. C., Cassinelli, J. P., Bjorkman, J. E., \& Lamers, H. J. G. L. M. 2000 ApJ, 534, 348

Lamers, H. J. G. L. M., Snow, T. P., \& Lindholm, D. M. 1995, ApJ, 455, 269

Lanz, T., \& Hubeny, I. 2003, ApJS, 146, 417

Limongi, M., \& Chieffi, A. 2006, ApJ, 647, 417

Lucy, L. B. 2007, A\&A, 468, 649 (L07)

Lucy, L. B. 2010, A\&A, 512, 33 (L10)

Lucy, L. B. \& Solomon, P. M. 1970, ApJ, 159, 879 (LS70)

Marcolino, W. L. F., Bouret, J.-C., Martins, F., et al. 2009, A\&A, 498, 837

Pols, O. R., Schroder, K.-P., Hurley, J. R., Tout, C. A., \& Eggleton, P. P. 1998, MNRAS, 298, 525

Smith, N., \& Conti, P. S. 2008, ApJ, 679, 1467

Vink, J. S., de Koter, A., \& Lamers, H. J. G. L. M. 2000, A\&A, 362, 295 